\title{
Specific bioelectrical impedance vector analysis (BIVA) is more accurate than classic BIVA to detect changes in body composition and in nutritional status in institutionalised elderly with dementia
}

\author{
$\mathrm{M}^{\mathrm{a} a}$. Alicia Camina Martín ${ }^{\mathrm{a}, *}$, Beatriz de Mateo Silleras a , Sara Barrera Ortega ${ }^{\mathrm{b}}$, \\ Luis Domínguez Rodríguez ${ }^{\mathrm{b}}, \mathrm{M}^{\mathrm{a}}$. Paz Redondo del Río ${ }^{\mathrm{a}}$ \\ a Area of Nutrition and Food Science, Faculty of Medicine, University of Valladolid, C/Ramón y Cajal 7, 47005 Valladolid, Spain \\ ${ }^{\mathrm{b}}$ Residential Care Centre San Juan de Dios, Psychogeriatric Area, Paseo Padre Faustino Calvo s/n Apto 66, 34005 Palencia, Spain
}

\section{A R T I C L E I N F O}

\section{Article history:}

Received 3 April 2014

Received in revised form 20 June 2014

Accepted 21 June 2014

Available online 25 June 2014

Section Editor: Holly M Brown-Borg

\section{Keywords:}

Specific BIVA

Classic BIVA

Nutritional assessment

Elderly

Dementia

\begin{abstract}
A B S T R A C T
Background: A new analytical variation of bioelectrical impedance vector analysis (BIVA), called specific BIVA, has shown to be more accurate in detecting changes in fat mass than classic BIVA.

Objective: To compare classic and specific BIVA in order to identify which is more strongly associated with psychofunctional and nutritional indicators in a group of institutionalised elderly patients with dementia.

Subjects and methods: Cross-sectional study. Fifty-four patients ( 34 women, 20 men) with dementia in moderately severe to very severe stages and aged 60-95 years underwent geriatric nutritional assessment, including body mass index calculations, the Mini Nutritional Assessment, the Geriatric Nutritional Risk Index, and whole body composition analysis.

Results: With specific BIVA (unlike with classic BIVA), significant differences were found between women with moderately severe and very severe dementia. In the BIVA conducted for body mass index, the confidence ellipses produced with the classic BIVA approach were highly overlapping; but with specific BIVA, significant differences were observed between the women in different nutritional categories (malnutrition, risk of malnutrition, normal weight and obesity). On the other hand, both approaches distinguished malnourished women from those who were at risk of malnutrition, according to the Mini Nutritional Assessment; and men with a moderate-high risk of malnutrition from men with no risk, on the basis of the Geriatric Nutritional Risk Index.

Conclusion: Overall, the findings of the present study suggest that specific BIVA is more effective than classic BIVA in identifying bioelectrical changes associated with psycho-functional and nutritional indicators in institutionalised elderly with dementia.
\end{abstract}

(C) 2014 Elsevier Inc. All rights reserved.

\section{Introduction}

The prevalence of malnutrition in institutionalised patients with dementia has been observed to be above 50\% (Faxén-Irving et al., 2005). However, it should be noted that there is also a high prevalence of undiagnosed subclinical malnutrition. This is of particular interest in this disease, since recent research has associated abdominal adiposity and high values of BMI in adulthood with an increased risk of developing

Abbreviations: AD, Alzheimer's disease; BIVA, bioelectrical impedance vector analysis; BMI, body mass index; DXA, dual-energy X-ray absorptiometry; ECW, extracellular water; ESPEN, European Society for Clinical Nutrition and Metabolism; GDS, Global Deterioration Scale; GNRI, Geriatric Nutritional Risk Index; ICW, intracellular water; MNA, Mini Nutritional Assessment; PA, phase angle; R, resistance; R sp, resistivity; SEGG, Sociedad Española de Geriatría y Gerontología; SGA, Subjective Global Assessment; Xc, reactance; Xc sp, reactivity; $Z$, impedance.

* Corresponding author at: Facultad de Medicina, Área de Nutrición y Bromatología, C/Ramón y Cajal, 7, 47005 Valladolid, Spain.

E-mail address: aliciacamina@gmail.com (Ma‥ A. Camina Martín). dementia in old age (Gustafson, 2012; Misiak et al., 2012). Indeed, changes in body composition (especially muscle mass depletion) are often masked by normal values of BMI. For these reasons, patients with dementia should undergo nutritional assessment and regular monitoring.

In clinical practice, geriatric nutritional assessment usually includes nutritional screening, a simple anthropometric assessment, measurement of various biochemical parameters, such as serum-albumin, and sometimes (but not always) body composition analysis. Among the nutritional screening tools available, the Mini Nutritional Assessment (MNA) is the most widely used in the geriatric population. It is also recommended by ESPEN for use in this group (Kondrup et al., 2003).

Regarding anthropometric assessment, the nutritional indicators normally used such as the BMI or the percentage of unintentional weight loss, are based on height and weight measurements. There are also indicators that combine nutritional and anthropometric parameters, such as the Geriatric Nutritional Risk Index (GNRI). This index includes the serum albumin level and the percentage of ideal weight, 
and it has proven to be a good predictor of muscle dysfunction (Cereda and Vanotti, 2005), morbidity and mortality in institutionalised elderly individuals (Cereda et al., 2008, 2011).

Finally, for the analysis of body composition, techniques are needed which are non-invasive, affordable, safe and simple, requiring the minimum possible collaboration by the elderly patient. The bioelectrical impedance analysis meets all these criteria and it has been considered by the European Working Group on Sarcopenia in Older People to be a good alternative to dual energy X-ray absorptiometry (DXA) for body composition analysis in older people (Cruz-Jentoft et al., 2010).

Bioelectrical impedance analysis allows volumes of body compartments to be estimated from multiple regression equations. However, the use of predictive equations requires assumptions to be made about body compartments, such as there being no fluid imbalance or body shape abnormalities, and these conditions have been found to be violated in elderly institutionalised individuals (Norman et al., 2007). In contrast, the vectorial approach to bioelectrical impedance analysis, called bioelectrical impedance vector analysis (BIVA), does not yield any absolute estimate of body compartments, makes no assumptions about body geometry, hydration state, or the electrical model of cell membranes, and is unaffected by regression adjustments (Nescolarde, 2006). In the BIVA approach, soft tissues, hydration status and cell integrity are assessed with a resistance-reactance ( $\mathrm{R}-\mathrm{Xc}$ ) graph, using the two direct components of the impedance vector (resistance and reactance, at $50 \mathrm{kHz}$ and $800 \mu \mathrm{A})$ standardised by height.

The BIVA method is valid for nutritional assessment both in healthy (Buffa et al., 2009) and pathological (Norman et al., 2007) aged populations. Comparing BIVA with DXA for body composition analysis in elderly individuals, it has been found that BIVA is useful for detecting variations in muscle mass but is less effective for identifying variations in fat mass (Buffa et al., 2013; Marini et al., 2013). In this regard, a new analytical variation of the method, called specific BIVA, has shown to be more accurate in detecting changes in fat mass than classic BIVA (Buffa et al., 2013; Marini et al., 2013). In specific BIVA (BIVA sp), the individual values for $\mathrm{R}$ and $\mathrm{Xc}$ are converted to resistivity (or specific resistance, $\mathrm{R} s p$ ) and reactivity (or specific reactance, $\mathrm{Xc} s p$ ) by adjusting for the length and cross-sectional area of the body. In addition, $\mathrm{R} s p$ and Xc $s p$ of the whole body (total R $s p$ and total Xc $s p$ ) are calculated assuming that the arms account for $45 \%$, the legs for $45 \%$, and the trunk for 10\% of the total R sp and Xc sp (Buffa et al., 2013).

Several studies have examined the relationship between the impedance vector analysis performed with the classic approach and various nutritional indicators, including the MNA and the Subjective Global Assessment (SGA), and significant associations have been observed (Buffa et al., 2009; Norman et al., 2007, 2008). However, there have been no previous reports of comparisons with specific BIVA.

The aim of this study was to compare the two BIVA approaches (classic and specific) in order to identify which is more strongly associated with Global Deterioration Scale (GDS) and MNA scores, BMI, and GNRI in a group of institutionalised elderly patients with dementia.

\section{Subjects and methods}

\subsection{Subjects}

Elderly patients institutionalised in the Psychogeriatric Area of the Residential Care Centre San Juan de Dios (Palencia, Spain) were recruited for the study. Inclusion criteria were: Caucasian ethnicity, BMI between 16 and $34 \mathrm{~kg} / \mathrm{m}^{2}$, and diagnosis of dementia according to the DSM IV criteria (American Psichiatric Association, 1997) in moderately-severe to very severe stages, according to the GDS scale (GDS 5, 6 and 7, respectively) (Reisberg et al., 1982). Patients were excluded if they had ongoing acute illness, or had pacemakers or metal implants.

Finally, 54 patients participated in the study, 20 (19.6\% of patients) men (mean, 95\% CI age: 76.5, 71.9-81.0 y; range: 60-92 y) and 34 (33.3\% of patients) women (mean, 95\% CI age: $82.1,79.2-84.9$ y; range: $60-95$ y). This study was conducted according to the guidelines laid down in the Declaration of Helsinki and all procedures involving human subjects/patients were approved by the Ethics Committee of the centre. Written informed consent was obtained from all subjects or their legal guardians.

\subsection{Methods}

Data on nutritional parameters and indicators used in routine geriatric nutritional assessment were recorded for all patients, assessments being completed within a single day for each individual. Anthropometric and whole body impedance measurements were taken first thing in the morning (following an overnight fast). Then, the MNA full-form was completed and GNRI was calculated.

\subsubsection{Mini Nutritional Assessment (MNA)}

The MNA full-form (Vellas et al., 2006) was used, consisting of 18 point-weighted questions in four categories: anthropometry, global and dietary issues, and self-assessment.

The items related to self-assessment of health and nutritional status were rated with the lowest score corresponding to patients unable to answer due to cognitive impairment. The items concerning dietary intake were completed on the basis of information provided by auxiliary nurses.

\subsubsection{Geriatric Nutritional Risk Index (GNRI)}

GNRI was calculated and interpreted following the procedure described by Bouillane et al. (2005). Serum albumin (necessary to calculate the GNRI) was determined using standard methods (Tietz, 1986).

\subsubsection{Anthropometry}

Anthropometric measurements were taken in accordance with the SENPE and SEGG protocol (Wanden-Berghe, 2006). Body weight $(\mathrm{W}, \mathrm{kg}$ ) was measured, to the nearest $100 \mathrm{~g}$, using a SECA 954 chair scale with the subject in underwear; and height $(\mathrm{H}, \mathrm{m})$ was estimated from a knee height measurement with the equation of (Chumlea et al., 1985).

BMI was calculated as weight $(\mathrm{kg})$ divided by height squared $\left(\mathrm{m}^{2}\right)$. Patients were classified into five categories by BMI cut-offs established for elderly individuals (Wanden-Berghe, 2006): malnutrition $\left(<18.5 \mathrm{~kg} / \mathrm{m}^{2}\right)$, risk of malnutrition $\left(18.5-21.9 \mathrm{~kg} / \mathrm{m}^{2}\right)$, normal weight $\left(22-26.9 \mathrm{~kg} / \mathrm{m}^{2}\right)$, overweight $\left(27-29.9 \mathrm{~kg} / \mathrm{m}^{2}\right)$ and obesity $\left(>30 \mathrm{~kg} / \mathrm{m}^{2}\right)$.

Triceps, biceps, subscapular and suprailiac skinfold-thickness measurements were taken on the nondominant side of the body, with a Holtain constant pressure $\left(10 \mathrm{~g} / \mathrm{m}^{2}\right.$ ) calliper (to the nearest $0.2 \mathrm{~mm}$ ). Arm, waist and calf circumferences were measured with a flexible, inelastic measuring tape (to the nearest $1 \mathrm{~cm}$ ).

\subsubsection{Bioelectrical impedance vector analysis (BIVA)}

The bioelectrical values of resistance $(\mathrm{R}, \mathrm{Ohm})$ and reactance (Xc, Ohm) were obtained with a single-frequency impedance analyser (BIA 101, Akern, Florence, Italy) using an operating frequency of $50 \mathrm{kHz}$ and applying a current of $800 \mu \mathrm{A}$. Whole body impedance measurements were taken in accordance with the protocol described by Lukaski (1991). Phase angle (PA, degrees) and impedance (Z, Ohm) were calculated from $R$ and $X c$ as $\arctan X c / R$ and $\left(R^{2}+X c^{2}\right)^{0.5}$, respectively.

In order to assess which of the two BIVA methods (classic or specific) is more powerful to detect differences between subgroups obtained considering the BMI, GNRI and GDS and MNA scores, 95\% confidence ellipses were plotted for men and women. Direct impedance measurements, $\mathrm{R}$ and $\mathrm{Xc}$, were standardised by the subject's height (classic $\mathrm{BIVA}, \mathrm{R} / \mathrm{H}$ and $\mathrm{Xc} / \mathrm{H}, \mathrm{Ohm} / \mathrm{m}$ ) and by cross-sections of the body together with the height (specific BIVA, R sp and Xc sp, Ohm*cm), following the procedure described by Buffa et al. (2013). 


\subsubsection{Statistical analysis}

Statistical analysis was carried out using the SPSS ${ }^{\circledR}$ version $18 \cdot 0$ (SPSS, Chicago, IL, USA). All data are presented as mean (95\% CI) or median (5th-95th percentiles). The normality of the distribution of the variables was checked by the Kolmogorov-Smirnov test, and the homogeneity of variances by Levene's test.

Multiple comparisons were performed by ANOVA or Kruskal-Wallis tests, and post-hoc analysis by the Bonferroni test. A t-test or U de Mann Whitney test was used for pairwise comparisons, and correlation analyses were performed with Pearson or Spearman correlation tests. The level for significance was set at $\mathrm{p}<0.05$.

Vector analyses (classic and specific) were performed with BIVA software developed by Piccoli and Pastori (2002). Statistically significant differences between the mean vectors were assessed with the Hotelling's $\mathrm{T}^{2}$ test for vector analysis, and distance between groups with Mahalanobis distance.

\section{Results}

Table 1 shows the subjects stratified by BMI, GNRI, and MNA and GDS scores, as they have been presented in the BIVA plots. Most of the study sample (both men and women) had severe to very severe dementia. According to the BMI, the majority in both sexes had a normal weight, while the MNA scores indicate that $80 \%$ of the men and $60.6 \%$ of the women were at risk of malnutrition. On the basis of the GNRI, the risk of malnutrition was mostly moderate in both groups.

The anthropometric and bioelectric values are shown in Tables 2 and 3 , and the correlation between bioelectrical variables and indicators used in the nutritional assessment in Table 4. Triceps, biceps and subscapular skinfolds were significantly higher in the women, as were resistance ( $R, R / H$ and $R s p)$ and impedance ( $Z$ and $Z s p)$ values. Phase angle (PA) was significantly higher in the men (Table 3 ). No significant differences were found in any of the other variables analysed. In relation to the correlation analyses, generally, all the nutritional indicators were more strongly correlated with the bioelectric parameters standardised by cross-sections of the body and also height ( $\mathrm{R} s p, \mathrm{Xc} s p$ and $\mathrm{Z} s p$ ) than with bioelectrical parameters normalized only by height $(R / H$, $\mathrm{Xc} / \mathrm{H}$ and $\mathrm{Z} / \mathrm{H}$ ) or the raw bioelectric parameters (R, Xc and Z) (Table 4).

Confidence ellipses for the GDS subgroups are represented in Fig. 1. Neither classic nor specific BIVA was useful for distinguishing subgroups of men. On the other hand, with specific BIVA, significantly differences were found between women with moderately-severe and very severe dementia $\left(\mathrm{T}^{2}=8.0, \mathrm{p}=0.041, \mathrm{D}=1.37\right)$, while no significant

Table 1

Distribution of the study sample according to the groups used to draw the confidence ellipses.

\begin{tabular}{lcc}
\hline & Men $(\mathrm{n}=20)$ & Women $(\mathrm{n}=34)$ \\
\hline Global Deterioration Scale (GDS) scores & & \\
GDS 5 & $5(25.0)$ & $6(17.6)$ \\
GDS 6 & $8(40.0)$ & $13(38.2)$ \\
GDS 7 & $7(35.0)$ & $15(15.0)$ \\
Body Mass Index (BMI, kg/m²) & & \\
Malnutrition $(<18.5)$ & $0(0.0)$ & $5(14.7)$ \\
Risk of malnutrition $(18.5-21.9)$ & $7(35.0)$ & $6(17.6)$ \\
Normal weight $(22-26.9)$ & $13(65.0)$ & $18(52.9)$ \\
Overweight $(27-29)$ & $0(0.0)$ & $0(0.0)$ \\
Obesity (>30) & $0(0.0)$ & $5(14.7)$ \\
Mini Nutritional Assessment (MNA) & & \\
Malnutrition $(<17)$ & $4(20.0)$ & $13(39.4)$ \\
Risk of malnutrition $(17-23.5)$ & $16(80.0)$ & $20(60.6)$ \\
Normal $(\geq 24)$ & $0(0.0)$ & $0(0.0)$ \\
Geriatric Nutritional Risk Index (GNRI) & & \\
High Risk $(<82)$ & $2(10.0)$ & $5(14.7)$ \\
Moderate risk $(82-92)$ & $11(55.0)$ & $15(44.1)$ \\
Low risk $(92-98)$ & $5(25.0)$ & $6(17.6)$ \\
No risk $(>98)$ & $2(10.0)$ & $8(23.5)$
\end{tabular}

Results are expressed as absolute and relative frequencies: $\mathrm{n}(\%)$.
Table 2

Anthropometric variables by sex.

\begin{tabular}{lcc}
\hline & Men $(\mathrm{n}=20)$ & Women $(\mathrm{n}=34)$ \\
\hline Weight $(\mathrm{kg})$ & $60.75(56.97-64.52)^{*}$ & $53.45(49.33-57.58)$ \\
Height $(\mathrm{m})$ & $1.65(1.63-1.67)^{* * *}$ & $1.50(1.47-1.53)$ \\
AC $(\mathrm{cm})$ & $25.60(24.53-26.68)$ & $26.27(25.00-27.54)$ \\
WC $(\mathrm{cm})$ & $92.41(88.33-96.49)$ & $89.93(85.35-94.51)$ \\
CC $(\mathrm{cm})$ & $34.44(32.36-36.52)$ & $33.84(31.52-36.16)$ \\
TS $(\mathrm{mm})$ & $12.00(7.00-24.95)^{* *}$ & $21.55(19.89-24.21)$ \\
BS $(\mathrm{mm})$ & $7.42(6.30-8.54)^{* *}$ & $14.07(11.49-16.66)$ \\
SS $(\mathrm{mm})$ & $15.48(12.91-18.05)$ & $16.48(14.00-18.96)$ \\
SiS $(\mathrm{mm})$ & $13.00(9.54-16.46)^{* *}$ & $20.91(17.31-24.51)$ \\
\hline
\end{tabular}

AC, arm circumference $(\mathrm{cm})$; WC, waist circumference $(\mathrm{cm})$; CC, calf circumference $(\mathrm{cm})$; TS, triceps skinfold ( $\mathrm{mm})$; BS, biceps skinfold (mm); SS, subscapular skinfold (mm); SiS suprailiac skinfold ( $\mathrm{mm}$ )

Median values were significantly different from those of the women: ${ }^{*} P<0.05,{ }^{* *} P<0.01$, ${ }^{* * *} P<0.001$.

Results are expressed as mean (95\% CI) or median (P5-P95).

differences were observed when using classic BIVA $\left(T^{2}=4.2, p=0.165\right.$, $\mathrm{D}=0.99$ ).

In the BIVA stratifying by BMI (Fig. 2), significant differences were observed only among the women. The confidence ellipses produced with the classic BIVA approach were highly overlapping, and significant differences were observed only between normal weight and obese women $\left(\mathrm{T}^{2}=8.2, \mathrm{p}=0.036, \mathrm{D}=1.45\right)$. In contrast, when using specific BIVA, a positive relationship was observed between BMI and the values of R $s p$ and Xc $s p$. Significant differences were observed between women who were: malnourished and normal weight $\left(\mathrm{T}^{2}=19.9\right.$, $\mathrm{p}=0.001, \mathrm{D}=2.26)$, malnourished and obese $\left(\mathrm{T}^{2}=38, \mathrm{p}=0.001\right.$, $\mathrm{D}=3.9)$, at risk of malnutrition and obese $\left(\mathrm{T}^{2}=34.2, \mathrm{p}=0.001, \mathrm{D}=\right.$ $3.54)$, and normal weight and obese $\left(T^{2}=17.9, \mathrm{p}=0.002, \mathrm{D}=2.14\right)$.

With regard to the confidence ellipses for subgroups defined by MNA findings (Fig. 3), neither of the two BIVA approaches were able to distinguish between subgroups of men. However, both approaches distinguished malnourished women from those who were at risk of malnutrition (classic BIVA: $\mathrm{T}^{2}=14.1, \mathrm{p}=0.004, \mathrm{D}=1.34$, specific BIVA: $\mathrm{T}^{2}=18.4, \mathrm{p}=0.001, \mathrm{D}=1.53$ ).

Finally, the 95\% confidence ellipses for groups according to the GNRI are represented in Fig. 4. Both classic and specific BIVA approaches evidenced significantly differences between men without risk and men with moderate-to-high risk (classic BIVA: $\mathrm{T}^{2}=10.5, \mathrm{p}=0.019, \mathrm{D}=$ 1.42; specific BIVA: $\mathrm{T}^{2}=11.3, \mathrm{p}=0.014, \mathrm{D}=1.49$ ). In females, significant differences were observed when comparing the subgroup without nutritional risk with the following subgroups: low risk (specific BIVA: $\mathrm{T}^{2}=13.6, \mathrm{p}=0.014, \mathrm{D}=1.99$ ), moderate risk (classic BIVA: $\mathrm{T}^{2}=$ $15.5, \mathrm{p}=0.004, \mathrm{D}=2.32$; specific BIVA: $\mathrm{T}^{2}=12.6, \mathrm{p}=0.009, \mathrm{D}=$ 1.55 ), and high risk (classic BIVA: $\mathrm{T}^{2}=13.4, \mathrm{p}=0.017, \mathrm{D}=0.59$;

Table 3

Bioelectrical variables by sex.

\begin{tabular}{lcc}
\hline & Men $(\mathrm{n}=20)$ & Women $(\mathrm{n}=34)$ \\
\hline $\mathrm{R}(\Omega)$ & $565.95(531.35-600.55)^{*}$ & $623.15(596.37-649.92)$ \\
$\mathrm{Xc}(\Omega)$ & $39.15(35.83-42.47)$ & $38.59(35.57-41.61)$ \\
$\mathrm{Z}(\Omega)$ & $567.34(532.74-601.95)^{*}$ & $624.39(597.58-651.20)$ \\
$\mathrm{PA}($ degrees$)$ & $3.98(3.65-4.31)^{*}$ & $3.55(3.30-3.79)$ \\
$\mathrm{R} / \mathrm{H}(\Omega / \mathrm{m})$ & $350.42(326.90-373.95)^{* * * *}$ & $417.46(396.50-418.42)$ \\
$\mathrm{Xc} / \mathrm{H}(\Omega / \mathrm{m})$ & $24.23(22.12-26.33)$ & $25.81(23.73-27.89)$ \\
$\mathrm{Z} / \mathrm{H}(\Omega / \mathrm{m})$ & $351.29(327.75-374.82)^{* * * *}$ & $418.29(397.30-439.27)$ \\
$\mathrm{R} \mathrm{sp}(\Omega \times \mathrm{cm})$ & $455.54(397.28-733.70)^{*}$ & $562.81(521.33-604.28)$ \\
$\mathrm{Xc} \mathrm{sp}(\Omega \times \mathrm{cm})$ & $33.89(29.70-38.08)$ & $34.91(31.50-38.33)$ \\
$\mathrm{Z} \mathrm{sp}(\Omega \times \mathrm{cm})$ & $456.49(398.07-736.05)^{*}$ & $563.93(522.38-605.48)$ \\
\hline
\end{tabular}

R, resistance $(\Omega)$; Xc, reactance $(\Omega) ; Z$, impedance $(\Omega) ; \mathrm{R} / \mathrm{H}$, resistance standardised by height $(\Omega / \mathrm{m}) ; \mathrm{Xc} / \mathrm{H}$, reactance standardised by height $(\Omega / \mathrm{m}) ; \mathrm{Z} / \mathrm{H}$, impedance standardised by height $(\Omega / \mathrm{m})$; R sp, specific resistance $(\Omega \times \mathrm{cm}) ; \mathrm{Xc}$ sp, specific reactance $(\Omega \times \mathrm{cm}) ; \mathrm{Z} s p$, specific impedance $(\Omega \times \mathrm{cm})$.

Median values were significantly different from those of the women: ${ }^{*} P<0.05,{ }^{* *} P<0.01$, ${ }^{* * *} P<0.001$.

Results are expressed as mean (95\% CI) or median (P5-P95). 
Table 4

Correlation between bioelectrical variables and nutritional indicators by sex.

\begin{tabular}{|c|c|c|c|c|c|c|}
\hline & \multicolumn{3}{|l|}{ Men } & \multicolumn{3}{|l|}{ Women } \\
\hline & BMI & MNA & GNRI & BMI & MNA & GNRI \\
\hline $\mathrm{R}(\Omega)$ & -0.224 & -0.130 & -0.217 & $-0.526^{* *}$ & $-0.581^{* *}$ & $-0.631^{* *}$ \\
\hline $\operatorname{Xc}(\Omega)$ & -0.066 & 0.272 & 0.243 & -0.197 & 0.052 & -0.165 \\
\hline $\mathrm{Z}(\Omega)$ & -0.224 & -0.128 & -0.316 & $-0.526^{* *}$ & $-0.579^{* *}$ & $-0.630^{* *}$ \\
\hline PA (degrees) & 0.056 & 0.332 & 0.420 & 0.068 & $0.380^{*}$ & 0.161 \\
\hline $\mathrm{R} / \mathrm{H}(\Omega / \mathrm{m})$ & -0.051 & -0.141 & -0.206 & $-0.450^{* *}$ & $-0.507^{* *}$ & $-0.615^{* *}$ \\
\hline $\mathrm{Xc} / \mathrm{H}(\Omega / \mathrm{m})$ & 0.075 & 0.268 & 0.347 & -0.191 & 0.034 & -0.209 \\
\hline $\mathrm{Z} / \mathrm{H}(\Omega / \mathrm{m})$ & -0.050 & -0.139 & -0.206 & $-0.450^{* *}$ & $-0.505^{* *}$ & $-0.614^{* *}$ \\
\hline $\mathrm{R} s p(\Omega \times \mathrm{cm})$ & $0.688^{* *}$ & 0.310 & 0.125 & $0.783^{* *}$ & $0.432^{*}$ & $0.693^{* *}$ \\
\hline Xc sp $(\Omega \times \mathrm{cm})$ & $0.603^{* *}$ & $0.487^{*}$ & $0.555^{*}$ & $0.612^{* *}$ & $0.551^{* *}$ & $0.606^{* *}$ \\
\hline $\mathrm{Zsp}(\Omega \times \mathrm{cm})$ & $0.688^{* *}$ & 0.312 & 0.125 & $0.783^{* *}$ & $0.433^{*}$ & $0.693^{* *}$ \\
\hline
\end{tabular}

R, resistance $(\Omega)$; Xc, reactance $(\Omega) ; Z$, impedance $(\Omega) ; \mathrm{R} / \mathrm{H}$, resistance standardised by height $(\Omega / \mathrm{m}) ; \mathrm{Xc} / \mathrm{H}$, reactance standardised by height $(\Omega / \mathrm{m}) ; \mathrm{Z} / \mathrm{H}$, impedance standardised by height $(\Omega / \mathrm{m})$; R sp, specific resistance $(\Omega \times \mathrm{cm})$; Xc sp, specific reactance $(\Omega \times \mathrm{cm}) ; \mathrm{Z} s p$, specific impedance $(\Omega \times \mathrm{cm})$.

P-Pearson and Rho de Spearman: ${ }^{*} \mathrm{P}<0.05,{ }^{* *} \mathrm{P}<0.01$.

specific BIVA: $\left.\mathrm{T}^{2}=24.0, \mathrm{p}=0.003, \mathrm{D}=2.79\right)$; as well as comparing low and moderate risk (classic BIVA: $\mathrm{T}^{2}=17.9, \mathrm{p}=0.002, \mathrm{D}=$ 1.43 ), and low and high risk (classic BIVA: $\mathrm{T}^{2}=14.7, \mathrm{p}=0.018, \mathrm{D}=$ 1.72; specific BIVA: $\mathrm{T}^{2}=11.0, \mathrm{p}=0.037, \mathrm{D}=2.01$ ) groups.

\section{Discussion}

The clinical interpretation of the bioelectrical parameters using the $\mathrm{R}-\mathrm{Xc}$ graph is simple. The impedance vector length $(\mathrm{Z})$ and the phase angle (PA) are defined by resistance (R) and reactance (Xc) respectively. $\mathrm{R}$ is the opposition to flow of an alternating current through intraand extracellular ionic solutions, and it is inversely related to the intra- and extracellular water (ICW and ECW). On the other hand, Xc represents the additional opposition due to the capacitance of the cell membranes, and hence it is directly related to the amount of soft tissue structures (Piccoli and Pastori, 2002). Therefore, an individual with a long vector length and a small PA (high $\mathrm{R}$ and low Xc values) has a poorer nutritional and hydration status than another subject (same sex, age, and BMI) with similar vector length and higher PA, since vector length is influenced by tissue hydration (and thus fat free mass), and vector direction (PA) is influenced by the amount of cell mass contained in soft tissues (Bosy-Westphal et al., 2005; Piccoli and Pastori, 2002).

BIVA can be used to diagnose the nutritional and hydration status of individuals, plotting the point vector on the reference bivariate tolerance ellipses; and also to compare groups, using the bivariate $95 \%$ confidence ellipses of the mean vectors (Piccoli and Pastori, 2002). The reference bivariate tolerance ellipses currently available were developed with the classic BIVA approach, using the bioelectrical parameters standardised by height; and more recently with the specific BIVA

\section{MEN}
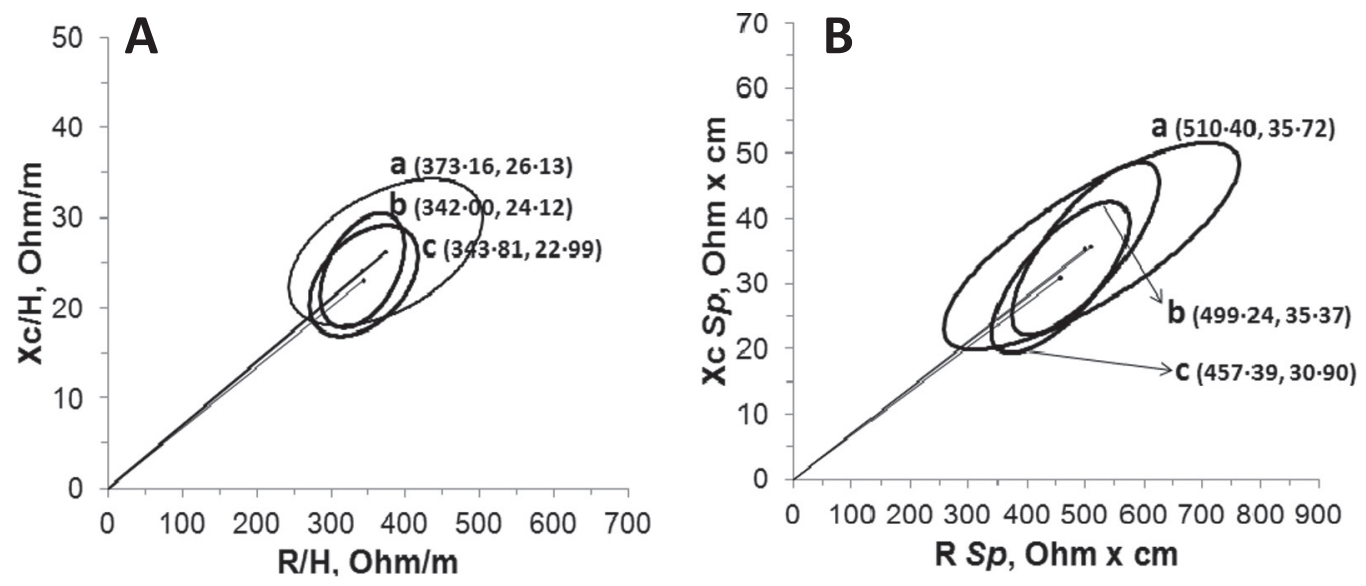

\section{WOMEN}
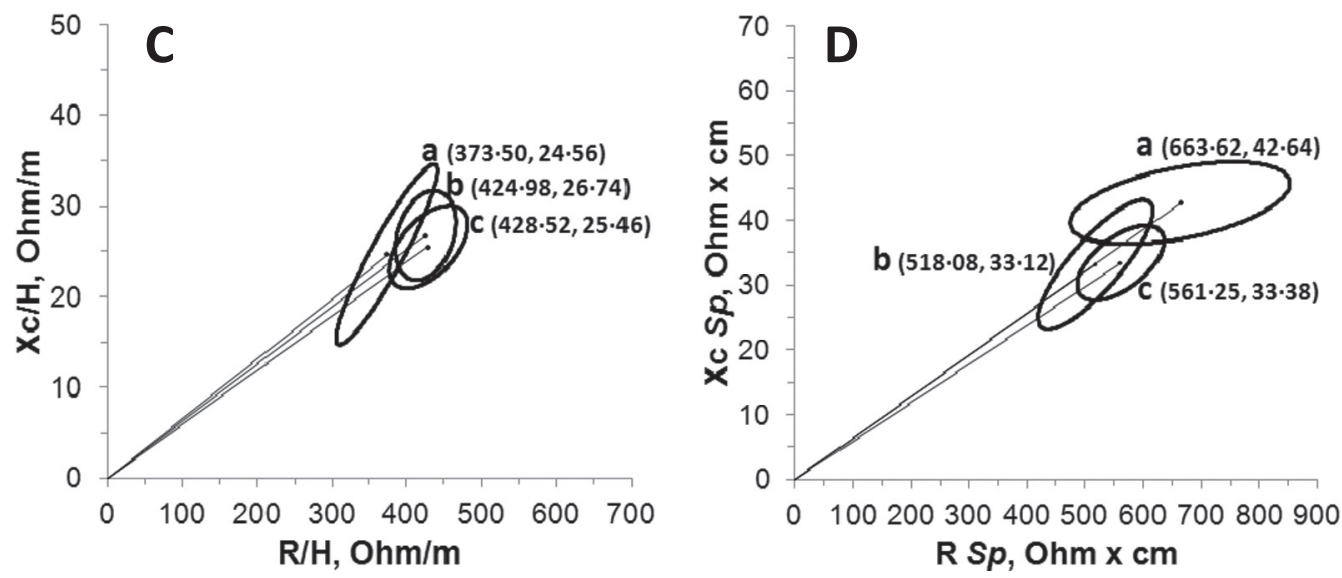

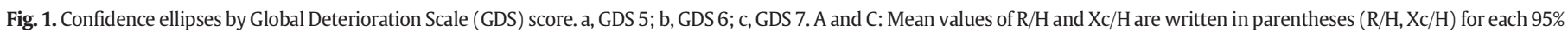
confidence ellipse. B and D: Mean values of R $s p$ and Xc $s p$ are written in parentheses ( $\mathrm{R} s p$, Xc $s p$ ) for each $95 \%$ confidence ellipse. 


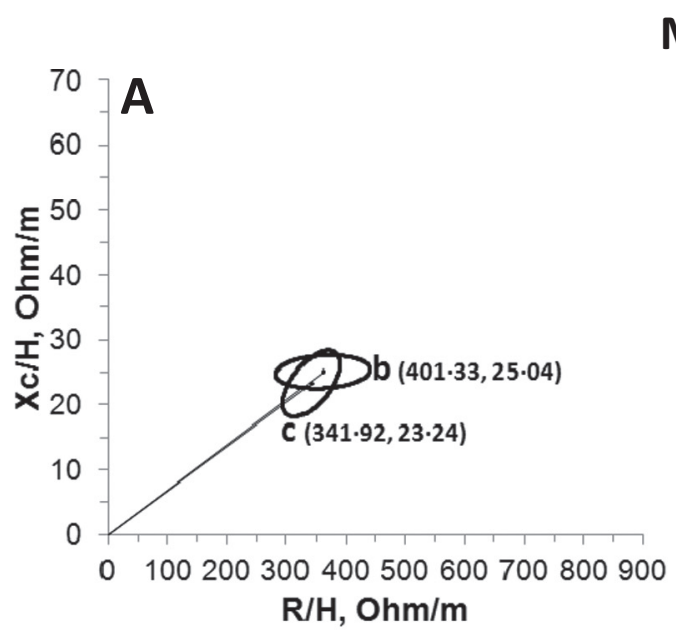

MEN

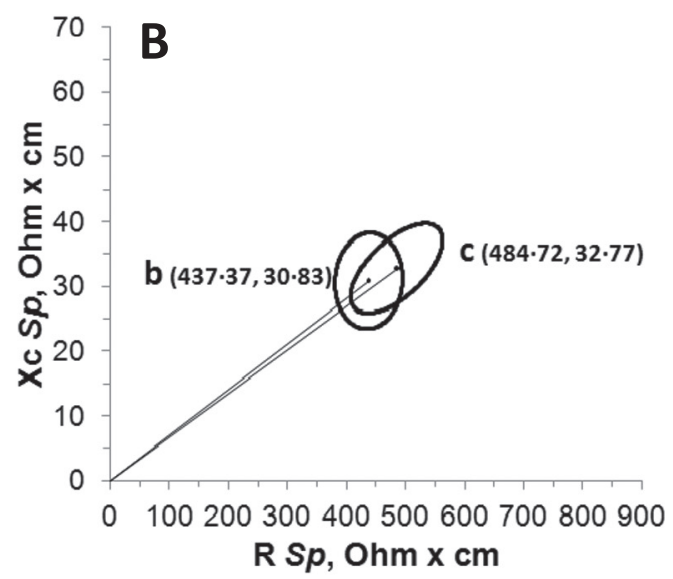

WOMEN
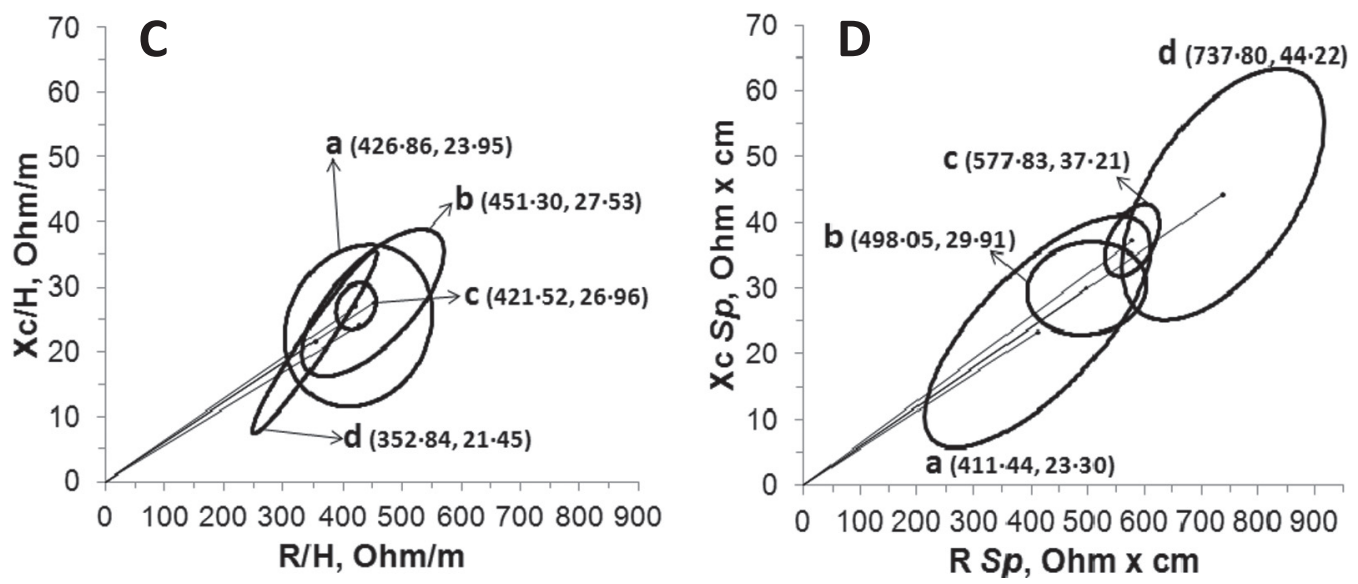

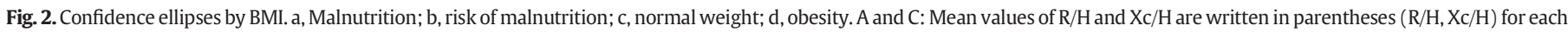
95\% confidence ellipse. B and D: Mean values of R $s p$ and Xc sp are written in parentheses (R $s p$, Xc $s p$ ) for each 95\% confidence ellipse.

approach, using the bioelectrical parameters standardised by crosssections of the body together with the height (Saragat et al., 2014). Their diagnostic efficacy has been evidenced in various clinical contexts, and it has been observed a good correlation between bioelectrical parameters and clinical diagnosis (Bronhara et al., 2012). In regard to the specific BIVA method, Buffa et al. (2013) compared the accuracy of the classic and specific BIVA approaches in a large multiethnic sample (1590 adult individuals aged 21-49 years), and they demonstrated that specific BIVA is more accurate than classic BIVA in the assessment of FM percentage and ECW/ICW analysed with DXA and bioimpedance spectroscopy, respectively, as well as in the classification of individuals at the 5th and 95th percentiles for both indicators. Furthermore, it was also recently reported that classic BIVA distinguishes between elderly individuals with different amounts of fat and fat-free mass (according to DXA), but not between those with different fat mass percentages (FM\%) (Marini et al., 2013).

In view of the findings of Buffa et al. (2013), and taking into account the demonstrated relationship between the bioelectrical patterns from classic BIVA and nutritional indicators routinely used in the geriatric nutritional assessment, we hypothesized that specific BIVA might also be more effective than classic BIVA when considering nutritional indicators. As hypothesized, the correlation analyses showed no significant correlations between any of the nutritional indicators (BMI, MNA score and GNRI) and $\mathrm{R} / \mathrm{H}$ or $\mathrm{Xc} / \mathrm{H}$ in the group of men, but there was a significant correlation between Xc sp and all the nutritional indicators and between R sp and BMI (Table 4). In the group of women, both R/H and $\mathrm{R} s p$ were significantly correlated with the three nutritional indicators, and also Xc sp (but not Xc/H) was correlated with the BMI, MNA scores and GNRI (Table 4). These results suggest that specific BIVA is more sensitive than classic BIVA for detecting changes in body cell mass, which is expected to be more affected than hydration status with changes in BMI, MNA score and GNRI.

In relation to the $95 \%$ confidence ellipses of the mean vectors of GDS scores, no differences were found in either men or women using classic BIVA. Nevertheless, the R/H-Xc/H graphs (Fig. 1, A and C) showed a tendency to a longer impedance vector and a smaller PA with progression of the disease, as has been previously found in patients with Alzheimer's disease (Buffa et al., 2010). In contrast, a significant mean vector displacement was observed between GDS 5 and GDS 7 in women when using specific BIVA: both vector length and PA were higher in GDS 5 than in GDS 7, and this was due to changes in R $s p$ and Xc $s p$ values (Fig. 1). Therefore, both hydration status, fat mass and body cell mass are altered in advanced stages of dementia, and it was possible to detect these changes earlier using specific BIVA. This finding is certainly of interest in clinical practice, since practical guidelines for the diagnosis and management of malnutrition in Alzheimer's disease (the most prevalent form of dementia) recommend monitoring these two properties in order to prevent dehydration and protein-energy malnutrition 


\section{MEN}
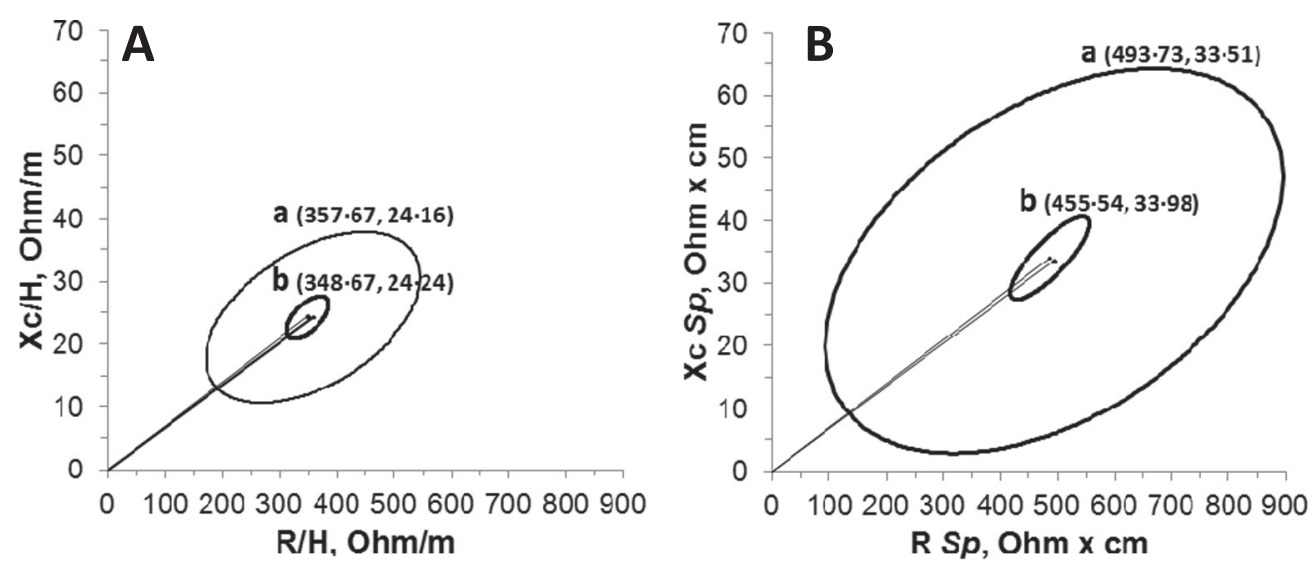

WOMEN
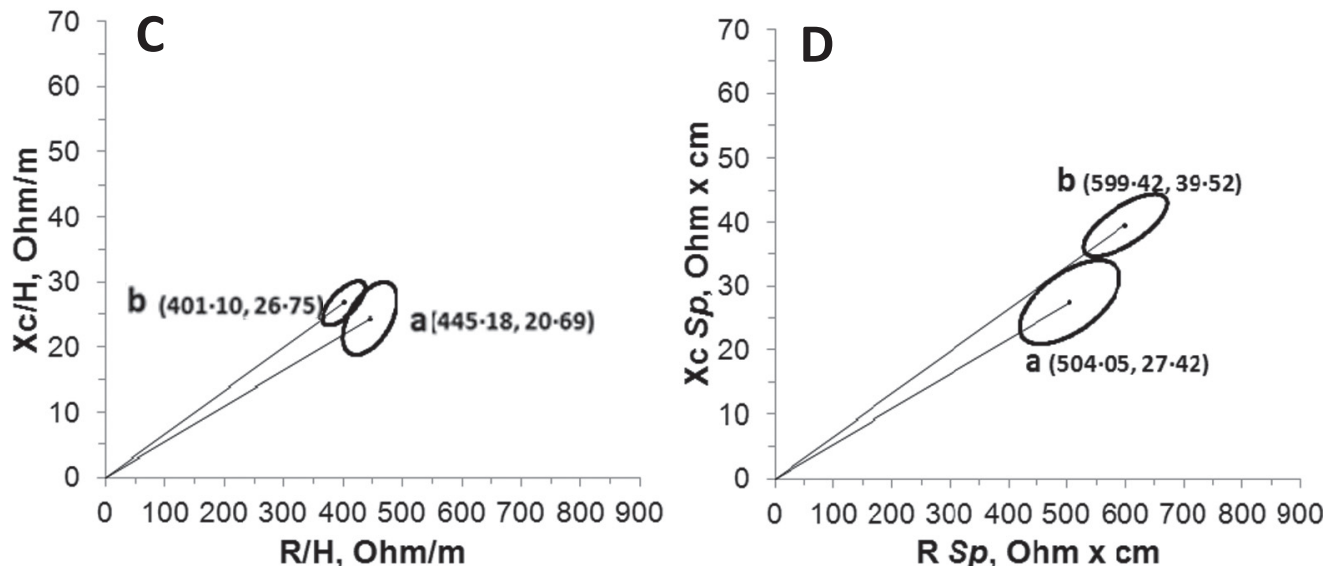

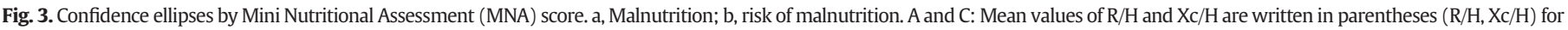
each 95\% confidence ellipse. B and D: Mean values of R $s p$ and Xc sp are written in parentheses (R $s p$, Xc sp) for each 95\% confidence ellipse.

(Belmin and Expert Panel and Organization Committee, 2007), which are very prevalent in demented patients (Alagiakrishnan et al., 2013; Saka et al., 2010).

Similar findings were obtained when using the 95\% confidence ellipses of the mean vectors of BMI, MNA score and GNRI in women (Figs. 2, 3 and 4 respectively). Both $\mathrm{R} s p$ and Xc $s p$ were directly associated with BMI, MNA score and GNRI. The mean impedance vector length shortened in parallel with the cataloguing achieved using each of the nutritional indicators; from obesity to malnutrition (in the BMI ellipses) and from no risk of malnutrition to malnutrition (in the GNRI and MNA ellipses). However, when employing the $\mathrm{R} / \mathrm{H}-\mathrm{Xc} / \mathrm{H}$ graphs, the significant mean vector displacements observed in all nutritional indicators were due to significant changes in $\mathrm{R} / \mathrm{H}$ values with comparable $\mathrm{Xc} / \mathrm{H}$ values; and, in contrast to specific BIVA, the mean vector lengths in all $\mathrm{R} / \mathrm{H}-\mathrm{Xc} / \mathrm{H}$ graphs were inversely related to BMI, MNA score and GNRI. These findings are consistent with the results of the correlation analyses (Table 4), and classic BIVA patterns were also similar to the findings of previous clinical research (Buffa et al., 2009; Norman et al., 2007; Santomauro et al., 2011). The discordances observed in the patterns of changes in the mean vector between classic and specific BIVA approaches were also expected. In relation to specific BIVA, there are currently no studies that examine the relationship between this method and nutritional indicators. Nevertheless, it is interesting to note that clinical research performed with segmental bioimpedance analysis has found a strong positive correlation between resistivity $(\mathrm{R} s p)$ and FM percentage (Biggs et al., 2001; Fuller et al., 2002), while height ${ }^{2} /$ impedance was higher and more positively correlated with total body water and FFM than with FM percentage (Fuller et al., 2002).

The PA values observed in the R-Xc graphs by BMI (Fig. 2, C and D) also were as expected. In the present study, the smallest PA value was observed in malnourished women, and well-nourished women had a higher mean PA value than the obese women using both BIVA approaches. Although this may seem paradoxical because low values of PA suggest poor nutritional status, an inverse correlation between BMI and PA has been found in severely obese subjects because of the increased ECW/ICW ratio of adipose tissue (Waki et al., 1991). Furthermore, it has been also found that obesity is associated with a poorer psycho-functional status in patients with AD (Saragat et al., 2012).

To our knowledge, this is the first clinical research in which specific BIVA has been applied to analyse bioelectrical changes associated with the routine nutritional and psycho-functional indicators used in geriatric nutritional assessment. The main limitation of the present study is the lack of a Gold Standard method for body composition analysis and the small size of the sample. The comparison of results obtained from specific BIVA with those obtained from a Gold Standard method would allow us to ensure the validity of our results. Regarding the sample size, it is well-known that the size of the confidence ellipses is influenced by sample size. Hence, finding significant differences in the confidence 


\section{MEN}
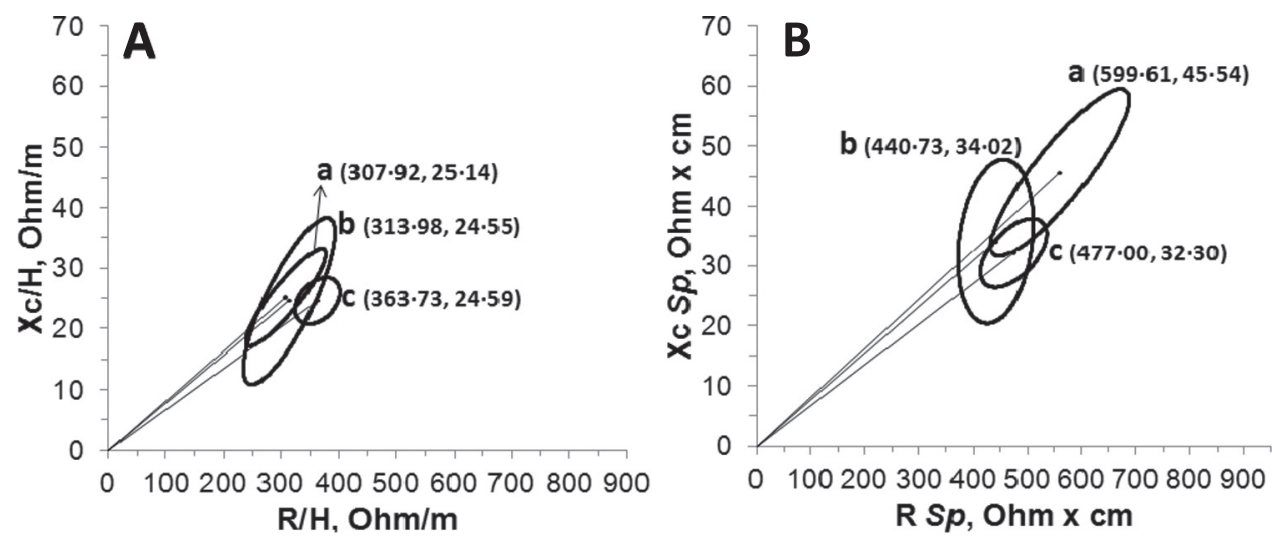

\section{WOMEN}
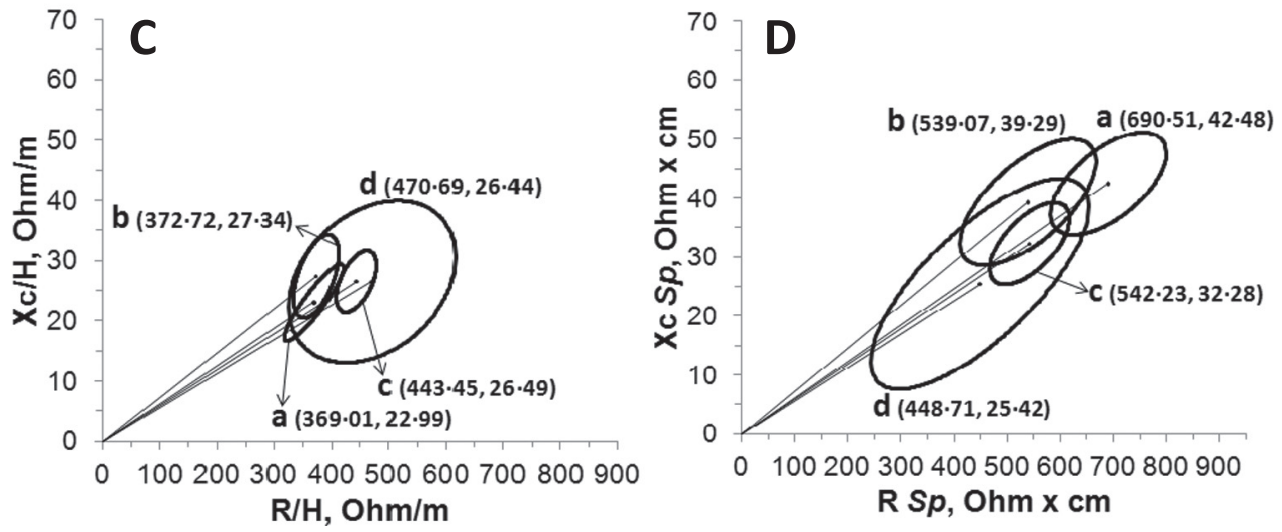

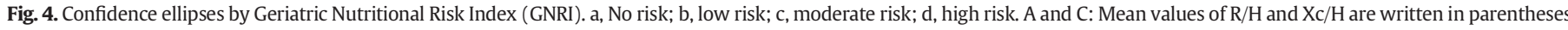
$(\mathrm{R} / \mathrm{H}, \mathrm{Xc} / \mathrm{H})$ for each $95 \%$ confidence ellipse. B and D: Mean values of R $s p$ and Xc sp are written in parentheses (R $s p$, Xc $s p$ ) for each 95\% confidence ellipse.

ellipses drawn for the group of men $(\mathrm{n}=20)$ is difficult, although the $\mathrm{R}-\mathrm{Xc}$ graphs do reflect the patterns noted in the group of women.

\section{Conclusion}

Specific BIVA is more effective than classic BIVA in identifying bioelectrical changes associated with GDS and MNA scores, BMI, and GNRI in institutionalised elderly individuals with dementia.

\section{Conflict of interest}

None.

\section{References}

Alagiakrishnan, K., Bhanji, R.A., Kurian, M., 2013. Evaluation and management of oropharyngeal dysphagia in different types of dementia: a systematic review. Arch. Gerontol. Geriatr. 56, 1-9.

American Psichiatric Association, 1997. Diagnostic and Statistical Manual of Mental Disorders, 4th edition. APA, Washington (DC) (DSM IV).

Belmin, J., Expert Panel and Organization Committee, 2007. Practical guidelines for the diagnosis and management of weight loss in Alzheimer's disease: a consensus from appropriateness ratings of a large expert panel. J. Nutr. Health Aging 11, 33-37.

Biggs, J., Cha, K., Horch, K., 2001. Electrical resistivity of the upper arm and leg yields good estimates of whole body fat. Physiol. Meas. 22, 365-376.

Bosy-Westphal, A., Danielzik, S., Dörhöfer, R.P., et al., 2005. Patterns of bioelectrical impedance vector distribution by body mass index and age: implications for bodycomposition analysis. Am. J. Clin. Nutr. 82, 60-68.

Bouillane, O., Morineau, G., Dupont, C., et al., 2005. Geriatric Nutritional Risk Index: a new index for evaluating at-risk medical patients. Am. J. Clin. Nutr. 82, 777-783.
Bronhara, B., Piccoli, A., Pereira, J.C., 2012. Fuzzy linguistic model for bioelectrical impedance vector analysis. Clin. Nutr. 31, 710-716.

Buffa, R., Floris, G., Marini, E., 2009. Assessment of nutritional status in free-living elderly individuals by bioelectrical impedance vector analysis. Nutrition 25, 3-5.

Buffa, R., Mereu, R.M., Putzu, P.F., et al., 2010. Bioelectrical impedance vector analysis detects low body cell mass and dehydration in patients with Alzheimer disease. J. Nutr. Health Aging 14, 823-827.

Buffa, R., Saragat, B., Cabras, S., et al., 2013. Accuracy of Specific BIVA for the Assessment of body composition in the United States Population. PLoS One 8, e58533.

Cereda, E., Vanotti, A., 2005. The new Geriatric Nutritional Risk Index is a good predictor of muscle dysfunction in institutionalized older patients. Clin. Nutr. 26 $78-83$.

Cereda, E., Zagami, A., Vanotti, A., et al., 2008. Geriatric Nutritional Risk Index and overallcause mortality prediction in institutionalised elderly: a 3-year survival analysis. Clin. Nutr. 27, 717-723.

Cereda, E., Pedrolli, C., Zagami, A., et al., 2011. Nutritional screening and mortality in newly institutionalized elderly: a comparison between the geriatric nutritional risk index and the mini nutritional assessment. Clin. Nutr. 30, 793-798.

Chumlea, W.C., Roche, A.F., Steinbaugh, M.L., 1985. Estimating stature from knee height for persons 60 to 90 years of age. J. Am. Geriatr. Soc. 33, 116-120.

Cruz-Jentoft, A.J., Baeyens, J.P., Bauer, J.M., et al., 2010. Sarcopenia: European consensus on definition and diagnosis: report of the European Working Group on sarcopenia in older people. Age Ageing 39, 412-423.

Faxén-Irving, G., Basun, H., Cederholmi, T., 2005. Nutritional and cognitive relationships and long-term mortality in patients with various dementia disorders. Age Ageing $34,136-141$.

Fuller, N.J., Fewtrell, M.S., Dewit, O., et al., 2002. Segmental bioelectrical impedance analysis in children aged 8-12 y: 1. The assessment of whole-body composition. Int. J Obes. Relat. Metab. Disord. 26, 684-691.

Gustafson, D.R., 2012. Adiposity and cognitive decline: underlying mechanisms. J. Alzheimers Dis. 30 (Suppl. 2), S97-S112.

Kondrup, J., Allison, S.P., Elia, M., et al., 2003. ESPEN guidelines for nutrition screening 2002. Clin. Nutr. 22, 415-421.

Lukaski, H.C., 1991. Assessment of body composition using tetrapolar impedance analysis In: Whitehead, R.G., Prentice, A. (Eds.), New Techniques in Nutritional Research. Academic Press, San Diego, pp. 303-315. 
Marini, E., Sergi, G., Succa, V., et al., 2013. Efficacy of specific bioelectrical impedance vector analysis (BIVA) for assessing body composition in the elderly. J. Nutr. Health Aging 17, 515-521.

Misiak, B., Leszek, J., Kiejna, A., 2012. Metabolic syndrome, mild cognitive impairment and Alzheimer's disease-the emerging role of systemic low-grade inflammation and adiposity. Brain Res. Bull. 89, 144-149.

Nescolarde, L.D., 2006. Segmental and whole body electrical impedance measurements in dialysis patients. (PhD Thesis) Universitat Politècnica de Catalunya, Spain.

Norman, K., Smoliner, C., Valentini, L., et al., 2007. Is bioelectrical vector analysis of value in the elderly with malnutrition and impaired functionality? Nutrition 23, 564-569.

Norman, K., Smoliner, C., Kilbert, A., et al., 2008. Disease-related malnutrition but not underweight by BMI is reflected by disturbed tissue properties in the bioelectrical impedance analysis. Br. J. Nutr. 100, 590-595.

Piccoli, A., Pastori, G., 2002. BIVA Software. Department of Medical and Surgical Sciences, University of Padova, Padova, Italy (available at E-mail:apiccoli@unipd.it).

Reisberg, B., Ferris, S.H., De León, M.J., et al., 1982. The Global Deterioration Scale for assessment of primary degenerative dementia. Am. J. Psychiatry 139, 1136-1139.
Saka, B., Kaya, O., Ozturk, G.B., et al., 2010. Malnutrition in the elderly and its relationship with other geriatric syndromes. Eur. J. Clin. Nutr. 29, 745-748.

Santomauro, F., Olimpi, N., Baggiani, L., et al., 2011. Bioelectrical impedance vector analysis and Mini Nutritional Assessment in elderly nursing home residents. J. Nutr. Health Aging 15, 163-167.

Saragat, B., Buffa, R., Mereu, E., Succa, V., et al., 2012. Nutritional and psycho-functional status in elderly patients with Alzheimer's disease. J. Nutr. Health Aging 16, 231-236.

Saragat, B, Buffa, R. Mereu, E De Rui, M. Coin, A Sergi, G, Marini, E 2014 Specific bioelectrical impedance vector reference values for assessing body composition in the Italian elderly. Exp. Gerontol. 50, 52-56.

Tietz, N.W., 1986. Fundamentals of Clinical Chemistry, 2nd ed. W.B. Saunders Co., Philadelphia.

Vellas, B., Villars, H., Abellan, G., et al., 2006. Overview of MNA® - its history and challenges. J. Nutr. Health Aging 10, 456-465.

Waki, M., Kral, J.G., Mazariegos, M., et al., 1991. Relative expansion of extracellular fluid in obese vs. nonobese women. Am. J. Physiol. 261, E199-E203.

Wanden-Berghe, C., 2006. Valoración Antropométrica. In: Planas, M. (Ed.), Valoración Nutricional en el Anciano. GalénitasNigra-Trea, Madrid, pp. 77-96. 